\title{
sensors
}

ISSN 1424-8220

(C) 2001 by MDPI

http://www.mdpi.net/sensors

\section{Determination of Active Components in Rhubarb by Cyclodextrin-modified Capillary Zone Electrophoresis}

\author{
Shang Xiaoyu and Yuan Zhuobin* \\ Department of Chemistry, Graduate School, The University of Science and Technology of China, \\ Academia Sinica, Beijing, 100039, P. R. China. Tel: +86-10-68224596 \\ *Author to whom correspondence should be addressed. E-mail: xiaoyshang@eyou.com
}

Received: 21 November 2001 /Accepted: 13 December 2001 / Published: 14 December 2001

\begin{abstract}
A cyclodextrin-modified capillary zone electrophoresis was developed for the determination of four bioactive components in Rhubarb. Effects of $\mathrm{pH}, \beta-\mathrm{CD}$ concentration and organic modifier content on the migration and separation of the components were studied. The four components were separated in the running buffer of $50 \mathrm{mmol} / \mathrm{L} \mathrm{NaOH}-$ $\mathrm{H}_{3} \mathrm{BO}_{3}$ (pH 10.7) containing $10 \mathrm{mmol} / \mathrm{L} \beta-\mathrm{CD}$ and $12.5 \% \mathrm{v} / \mathrm{v}$ ethanol. The analytical performance of the method was tested with respect to linearity response, precision and recovery. The determination of two commercial Rhubarb samples under the optimized conditions showed a satisfactory result.
\end{abstract}

Keywords: Cyclodextrin-modified capillary zone electrophoresis; Rhubarb; Anthraquinone derivatives

\section{Introduction}

Rhubarb is an ancient herbal medicine. In traditional Chinese therapy, it is widely used for the treatment of many diseases such as curing mental and renal disorders, removing blood statis and purging heat etc. Pharmacological tests have shown that the bioactivities of Rhubarb include purgation, analgesic effects, antibacterial, antitumor, antioxidant and antimutagenicity [1]. The pharmaceutically bioactive components in Rhubarb are hydroxyanthraquinone derivatives: emodin, chrysophanol, physcion, rhein, aloe-emodin and their glycosides. TLC [2] and HPLC [3,4] have been commonly used for the separation and determination of active components in Rhubarb. 
In recent years, capillary electrophoresis (CE) has been widely used in pharmaceutical industry. Micellar electrokinetic capillary chromatography (MECC) [5,6] and microemulsion electrokinetic chromatography (MEEKC) [7] have been developed for the separation of anthraquinone derivatives in Rhubarb. The former method used micelle as pseudo-stationary phase, while the latter used an oilwater microemulsion to improve the mobile phase. In comparison with MECC, MEEKC had a wider migration time window and a higher resolution, but the buffer system was comparatively complex. $\beta$ cyclodextrin (CD) is one of the most useful agents in separation science. Cyclodextrin-modified capillary zone electrophoresis (CD-CZE) is effective for the difficult separations [8]. It shows high separation selectivity and high efficiency. However, CD-CZE method has not been reported for the separation of anthraquinone derivatives in Rhubarb. In this paper, we developed a CD-CZE method for the separation of physcion, emodin, aloe-emodin and rhein in Rhubarb. The method is simple and rapid for the determination of the four components in Rhubarb.

\section{Experimental}

Materials and Reagents. Emodin, aloe-emodin, rhein and physcion were purchased from the National Institute for the Control of Pharmaceuticals and Biological Products. Their structures are shown in Fig.1. $\beta$-CD was bought from the Development Center of Special Chemical Reagents in North China. O-phthalic acid was purchased from Beijing Chemical Factory. Commercial Rhubarb samples were bought from local herbal market in Anguo. Boric acid, sodium hydroxide and ethanol were of analytical grade. Double distilled water was used for preparing solutions.<smiles>[R]c1cc(O)c2c(c1)C(=O)c1cc([R])cc(O)c1C2=O</smiles>

$$
\begin{array}{lll}
\text { Physcion } & \mathrm{R}_{1}=\mathrm{OCH}_{3} & \mathrm{R}_{2}=\mathrm{CH}_{3} \\
\text { Emodin } & \mathrm{R}_{1}=\mathrm{OH} & \mathrm{R}_{2}=\mathrm{CH}_{3} \\
\text { Aloe-emodin } & \mathrm{R}_{1}=\mathrm{H} \quad \mathrm{R}_{2}=\mathrm{CH}_{3} \mathrm{OH} \\
\text { Rhein } \quad \mathrm{R}_{1}=\mathrm{H} \quad \mathrm{R}_{2}=\mathrm{COOH} \\
\text { Chrysophanol } \quad \mathrm{R}_{1}=\mathrm{H} \quad \mathrm{R}_{2}=\mathrm{CH}_{3}
\end{array}
$$

Figure 1. Structures of five compounds in Rhubarb.

Equipment and Conditions. All separations were performed on a 1229 type HPCE Analyser system (Beijing Institute of New Technology) with an UV detector $(254 \mathrm{~nm})$. The capillary (50 $\mu \mathrm{m}$ i.d. $\times 45 \mathrm{~cm}$ ) was uncoated fused-silica. The temperature was kept at $25 \pm 1^{\circ} \mathrm{C}$. The applied voltage was $15 \mathrm{kV}$. Sample solutions were introduced by electro-migration at $10 \mathrm{kV}$ for $5 \mathrm{~s}$. The running buffer was $50 \mathrm{mmol} / \mathrm{L} \mathrm{NaOH}-\mathrm{H}_{3} \mathrm{BO}_{3}$ containing $10 \mathrm{mmol} / \mathrm{L} \beta-\mathrm{CD}$. The capillary was rinsed at the beginning of each day's use with $0.1 \mathrm{~mol} / \mathrm{L} \mathrm{NaOH}$ followed with distilled water and then running buffer. 
Sample preparation. A $0.5 \mathrm{~g}$ sample of Rhubarb powder was extracted in Soxhlet extractor with 15 $\mathrm{ml}$ of $2 \mathrm{~mol} / \mathrm{L} \mathrm{H}_{2} \mathrm{SO}_{4}$ and $70 \mathrm{ml}$ chloroform. The extraction was continued until the aqueous phase was almost colorless. Then the chloroform extract was evaporated to dryness with a water bath. The residue was dissolved in anhydrous ethanol. After addition of $50 \mu \mathrm{l}$ internal standard (I.S.) solution (o-phthalic acid), the extract was diluted to $5 \mathrm{ml}$ with running buffer. The solution was filtered through a $0.45 \mu \mathrm{m}$ membrane before injection.

Extraction recovery studies. Fixed amounts of pure physcion, emodin, aloe-emodin and rhein were added to a sample of Rhubarb of known contents respectively. After the mixtures were extracted, their concentrations were determined according to the above-mentioned method.

\section{Results and Discussion}

Comparison of CZE with CD-CZE for Separation of Bioactive Components in Rhubarb

Capillary zone electrophoresis (CZE) showed only one peak for the separation of four bioactive components in Rhubarb in $50 \mathrm{mmol} / \mathrm{L} \mathrm{NaOH}-\mathrm{H}_{3} \mathrm{BO}_{3}$ solution. The change of buffer concentration and $\mathrm{pH}$ could not significantly improve resolution. Thus, $\mathrm{CZE}$ of $\mathrm{NaOH}-\mathrm{H}_{3} \mathrm{BO}_{3}$ system could not separate these components. Upon addition of $\beta$-CD to the buffer, however, the result showed four peaks corresponded to the bioactive components in Rhubarb, indicating an improvement in resolution. The effect of $\mathrm{pH}, \beta-\mathrm{CD}$ concentration, organic modifier and applied voltage on the separation of CD-CZE were studied one by one in the following text.

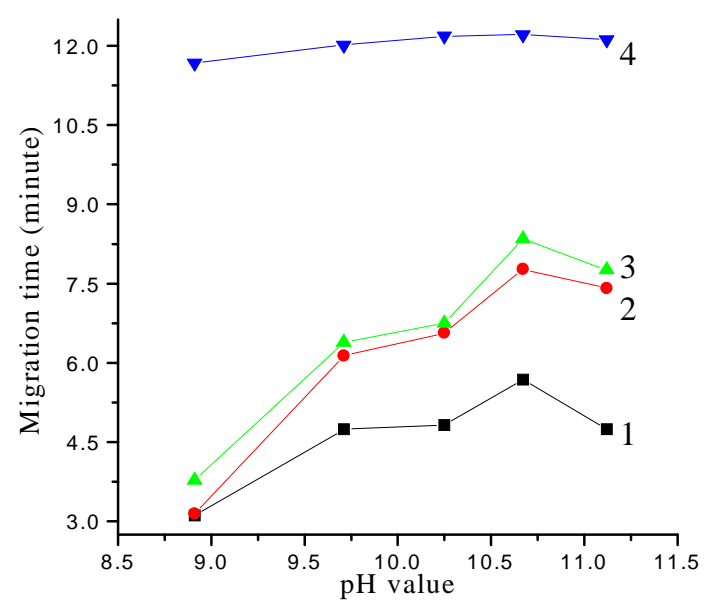

Figure 2. Effect of $\mathrm{pH}$ value on migration time.

1 - Physcion 2 -Emodin 3-Aloe-emodin 4-Rhein 


\section{Effect of $p H$}

The $\mathrm{pH}$ of running buffer is a key factor to selectivity of separation and affects the peak shape and resolution. In this experiment, high $\mathrm{pH}$ value was helpful to the separation due to the better solubilities of the four anthraquinone derivatives at high $\mathrm{pH}$. In the range of 8.91-11.12, the effect of $\mathrm{pH}$ on separation was shown in Fig.2. The migration times of the components varied greatly with the change of $\mathrm{pH}$, especially for that of physcion, emodin and aloe-emodin. Two factors affected the migration time: solubility and the electroosmotic flow (EOF). Both the increase in solubility and the decrease in EOF resulted in the increase of migration time. When $\mathrm{pH}$ was lower than 10.67 , the increase in solubilities of the compounds was the predominant factor, so the migration times of physcion, emodin and aloe-emodin increased with increasing $\mathrm{pH}$. When $\mathrm{pH}$ was higher than 10.67, increase of EOF was the primary factor, resulting in the reduction of migration times of the four compounds. In this range, rhein with one carboxylic group was completely ionized, so the migration time of rhein showed a little change. The best resolution was achieved by using the running buffer of $\mathrm{pH} 10.7$.

\section{Effect of $\beta$-CD concentration}

$\beta$-CD has a special cavity structure. The inside cavity is lipophilic, while the outside is hydrophilic. The lipophilic groups are able to get into the inside cavity of $\beta-C D$, resulting in the formation of hostguest complexes. Wang et.al [9] proved that anthraquinone derivatives could get into the cavity of $\beta$ $\mathrm{CD}$, so the inclusion-complexes of $\beta-\mathrm{CD}$ with the four compounds were formed upon addition of $\beta$ $\mathrm{CD}$. The different stability of these inclusion-complexes improved the separation selectivity. In the separation process, $\beta$-CD played two roles. Firstly, $\beta-C D$ acted as a pseudo-stationary phase. The four compounds showed different distribution between the pseudo-stationary phase and aqueous phase. On the other hand, it increased the solubilities of these components. Therefore $\beta-C D$ concentration was another important factor to the selectivity of separation. The migration times of the four compounds decreased with increasing of $\beta$-CD concentration from 2 to $13 \mathrm{mmol} / \mathrm{L}$. When $\beta$-CD concentration was more than $10 \mathrm{mmol} / \mathrm{L}$ or lower than $4 \mathrm{mmol} / \mathrm{L}$, the peaks of physcion, emodin and aloe-emodin overlapped together. In the $\beta$-CD concentration range of 4 and $10 \mathrm{mmol} / \mathrm{L} \beta-\mathrm{CD}$, the four components had the best separation selectivity. The representative electropherogram of the four components is drawn in Fig.3a.

\section{Influence of organic modifier}

Influence of anhydrous ethanol content on the separation was studied. Upon addition of anhydrous ethanol in the running buffer, migration times of the four components increased because of the decrease of the EOF, while the peak shape was improved. At an ethanol content of $12.5 \% \mathrm{v} / \mathrm{v}$, the four components were well separated. Large ethanol content increased solubilities of the four compounds in aqueous phase, but decreased the separating resolution. 


\section{Applied voltage}

At an applied voltage higher than $18 \mathrm{kV}$, separation time was reduced, and excessive Joule heating was produced as well. When applied voltage was lower than $15 \mathrm{kV}$, separation time was lengthened and separation efficiency was decreased. In the range of $15 \sim 18 \mathrm{kV}$, high separation efficiency could be achieved.
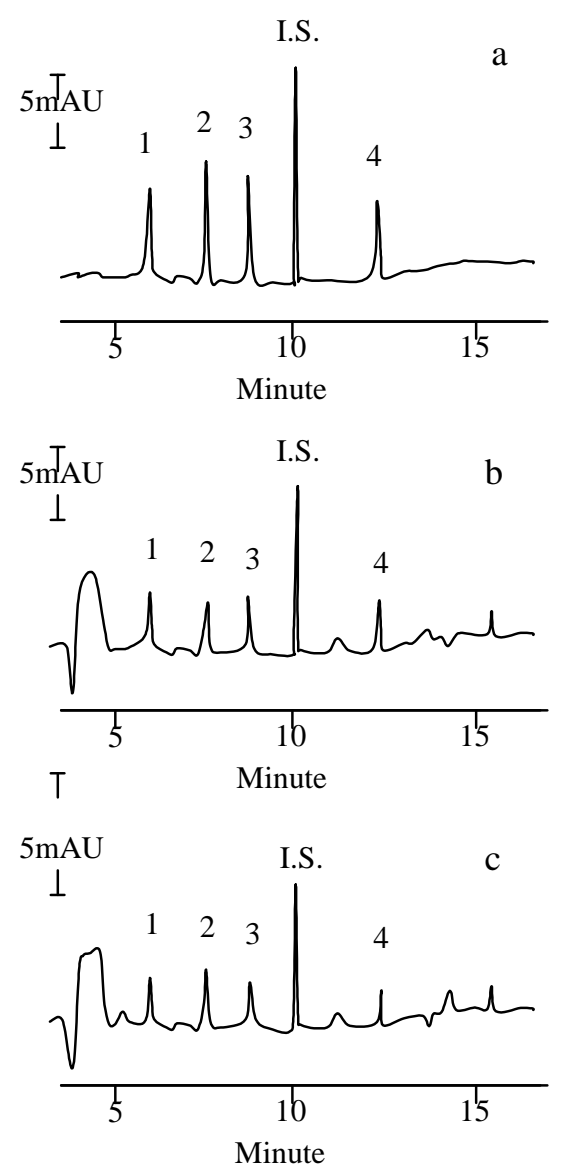

Figure 3. Typical electropherograms of standard samples and Rhubarb samples.

a) Standard samples b) Raw Rhubarb c) Mongolian Rhubarb. 1 physcion 2 emodin 3 aole-emodin 4 rhein

\section{Validation of the method}

The linearity of the four components in standard solutions was investigated in the range of 5- 60 $\mu \mathrm{g} / \mathrm{ml}$ for physcion, 4.8-58.2 $\mu \mathrm{g} / \mathrm{ml}$ for emodin, $4-32 \mu \mathrm{g} / \mathrm{ml}$ for aloe-emodin, and 4-40 $\mu \mathrm{g} / \mathrm{ml}$ for rhein. Calibration graphs were constructed in the linear range. The regression equations of curves and their correlation coefficients were calculated to be $y=11.36 x+0.0958$ ( 0.9991 , physcion); $y=13.20 x+0.1620$ (0.9994, emodin); $y=12.24 x-0.2011$ (0.9990, aloe-emodin) and $y=10.23 x-0.0012$ (0.9992, rhein). 
The reproducibility of this method was tested on the basis of peak-area (relative to I.S.) by measuring six replicate injections. For each component, the intra- and inter-day relative standard deviation (RSD) values were $1.87 \%$ and $2.33 \%$ for physcion, $1.56 \%$ and $1.92 \%$ for emodin, $1.71 \%$ and $2.08 \%$ for aloe-emodin and $1.62 \%$ and $2.15 \%$ for rhein.

The results of standard addition recovery of four components from sample composites of Rhubarb were calculated by the ratio of the amount produced and the amount added. The recovery was $92.6 \%$ for physcion, $100.01 \%$ for emodin, $98.7 \%$ for aloe-emodin and $97.2 \%$ for rhein.

\section{Determination of anthraquinone derivatives in Rhubarb samples}

Under the selected conditions, two samples of Raw Rhubarb and Mongolian Rhubarb were analyzed with the proposed method. The electropherograms are demonstrated in Fig.3b and Fig.3c respectively and the calculated contents of the anthraquinoids are listed in Table 1. No interference was observed in the two samples.

Table 1. Contents of anthraquinone derivatives in Rhubarb samples.

\begin{tabular}{|c|c|c|c|c|}
\hline \multirow{2}{*}{ Sample } & \multicolumn{4}{|c|}{ Content/mg g $^{-1}$} \\
\cline { 2 - 5 } & physcion & emodin & aloe-emodin & rhein \\
\hline Raw rhubarb & 1.157 & 1.035 & 1.129 & 1.016 \\
\hline Mongolian rhubarb & 0.893 & 1.317 & 1.098 & 0.932 \\
\hline
\end{tabular}

\section{Conclusions}

The established method in this work has successfully separated four anthraquinone derivatives in Rhubarb by optimizing the parameters such as $\mathrm{pH}, \beta-\mathrm{CD}$ concentration and organic modifier content. From the results of determination, the amounts of anthraquinone derivatives in Rhubarb samples were different from each other, therefore, the quality control of Rhubarb is necessary.

\section{Acknowledgements}

This work is supported by the National Natural Science Fundation of China (29675022, 29875027).

\section{References}

1. Chen, Q. H. The experimental study and application of Rhubarb. XinYiYaoXueZaZhi. 1974, 5, 3440.

2. He L.Y.; Luo Sh.R.. Analytical method study of anthraquinone derivatives in Chinese herbal drugs. Acta. Pharmaceutica Sinica. 1980, 15, 555-562. 
3. Ohshima, Y.; Ohno, Y.l Kajiyama, K.; Takahashi, K. High-performance liquid chromatographic separation of rhubarb constituents. J Chromatogr. 1986, 360, 303-306.

4. Kashiwada Y.; Nonaka G.; Nishioka I. Studies on Rhubarb (Rhbi Rhizoma). XV. Simultaneous Determination of Phenolic Constituents by High-performance Liquid Chromatography. Chem. Pharm. Bull. 1989, 37, 999-1004.

5. Zong, Y. Y.; Yu, M. T.; Zhu, ZH. D.; Che, ZH. T. Determiantion of Rhubarb by micellar electrokinetic capillary chromatography. Acta Pharmaceutica Sinica. 1995, 30, 594-598.

6. Sheu, Sh. J.; Chen, H. R. Determination of five major anthraquinoids in Chinese herbal preparations by micellar electrokinetic capillary electrophoresis. Analytica. Chimica. Acta. 1995, 309, 361-367.

7. Li, G. B.; Chen, X. G.; Liu, M. C.; Hu, Zh. D. Separation and identification of active components in the extract of Rheum natural products by microemulsion electrokinetic chromatography. Analyst. 1998, 123, 1501-1505.

8. Kumar, V. P.; Ross, B. L.; Samian, S. Enantiomeric and isomeric separation of herbicides using cyclodextrin-modified capillary zone electrophoresis. J Chromatogr. A, 1997, 790, 225-234.

9. Wang, B. Q.; Pang, ZH. G.; Li, Sh. Y. TLC of anthraquinone derivatives in Rhubarb. XianDaiYingYongYaoXue. 1993, 2, 19-21.

Sample Availability: Available from the author.

(C) 2001 by MDPI (http://www.mdpi.net). Reproduction is permitted for noncommercial purposes. 